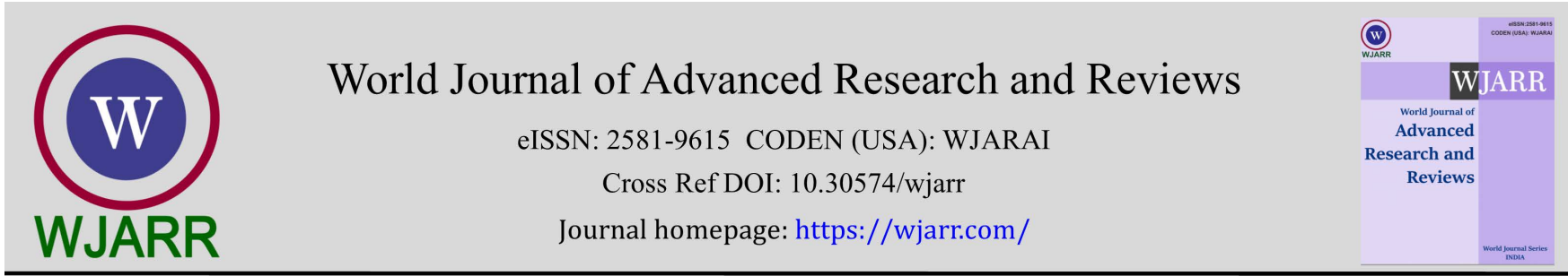

(CASE REPORT)

Check for updates

\title{
Abnormal night melatonin level in a patient with well-controlled epilepsy without sleep disturbances - could it be associated with levetiracetam therapy: A case report
}

\author{
Orlina Georgieva Chaneva ${ }^{1,2, *}$ and Zdravka Nikolaeva Vasileva 1,2 \\ ${ }^{1}$ Department of Neurology, Medical University - Plovdiv, 4000 Bulgaria 66 Pestersko shosse blvd. 4000 Plovdiv, Bulgaria. \\ ${ }^{2}$ Clinic of Neurology, UMHAT "St. George", Plovdiv, 4000 Bulgaria 66 Pestersko shosse blvd. 4000 Plovdiv, Bulgaria.
}

World Journal of Advanced Research and Reviews, 2021, 10(03), 085-087

Publication history: Received on 19 April 2021; revised on 06 June 2021; accepted on 10 June 2021

Article DOI: https://doi.org/10.30574/wjarr.2021.10.3.0239

\begin{abstract}
We present a case report of a 23-year old male with newly diagnosed epilepsy manifested with generalized tonic seizures. Levetiracetam (LEV) was started and the patient was seizure free. The patient demonstrated intermediate chronotype determined by the Morningness-Eveningness Questionnaire (MEQ). There was no epileptiform activity on electroencephalography (EEG) and polysomnography (PSG), sleep architecture disturbances (PSG) or daytime sleepiness (Epworth sleepiness score, Multiple sleep latency test) at the onset and after 3 months of LEV therapy. The melatonin serum levels after 3 months of LEV treatment were $22.00 \mathrm{pg} / \mathrm{ml}$ at $3 \mathrm{a} . \mathrm{m}$. and $23.60 \mathrm{pg} / \mathrm{ml}$ at 8 a.m. - there was no normal night peak concentration. This abnormality may be associated with a later night peak melatonin concentration, the treatment with levetiracetam being a possible explanation. We consider the presented clinical case of special interest because of the combination of absence of normal night peak melatonin concentration, normal sleep parameters and non-extremely presented chronotype. We suggest that such patients could benefit from add-on therapy with melatonin.
\end{abstract}

Keywords: Epilepsy; Seizures; Melatonin; Chronotype; Levetiracetam

\section{Introduction}

Sleep disorders and daytime sleepiness are common in patients with epilepsy [1-3]. The effect of epilepsy and sleep is reciprocal and it is controversial, whether the impaired sleep worsens seizure control, or the poor seizure control worsens sleep quality. On the other hand, it has been reported that sleep facilitates seizure activity in epilepsy [4]. Accumulating evidence suggests epilepsy and seizures may influence circadian rhythms and that circadian rhythms may influence epilepsy. It is also conceivable that seizure timing influences the timing of daily activities, sleeping, and wakefulness (i.e., chronotype) [5]. Some studies present the hypothesis that chronotype is related with epilepsy type. Patients with generalized epilepsy are more likely to be evening-type patients as compared to those with focal epilepsy or subjects without epilepsy. On the other hand, the data analysis shows some evidence that subjective chronotype in patients with epilepsy is more morning-oriented than in healthy controls [6, 7]. In addition to the sleep-wake cycle, the melatonin biorhythm synchronizes other cyclic processes such as the activity of the GABA-benzodiazepine receptor complex. Some research on melatonin levels during epileptic seizures support the hypothesis of the involvement of melatonin in the regulation of abnormal brain activity [8-11].

\footnotetext{
${ }^{*}$ Corresponding author: Orlina Georgieva Chaneva

Department of Neurology, Medical University - Plovdiv, 4000 Bulgaria 66 Pestersko shosse blvd. 4000 Plovdiv, Bulgaria. 


\section{Case report}

We present a case of a 23-year-old male with epilepsy onset with a generalized tonic seizure when he was 19 years old. He has had 3 more seizures with the same characteristics. For the next 3 years. After the fourth seizure the patient attended the Clinic of Neurology at the University Hospital in Plovdiv. All study procedures were performed after approval of the Local Ethics Commission at the Medical University, Plovdiv, Bulgaria. The patient was introduced to the study design and signed an informed consent form before participation in all study procedures. All medical history was collected by a trained neurologist specialized in epilepsy through an examination of the patients' medical documentation and a detailed interview on the disease.

Treatment with levetiracetam 2000 mg daily was started.

We present the patient's profile:

- newly diagnosed generalized epilepsy;

- $\quad$ severe generalized tonic seizures;

- $\quad$ structural etiology of epilepsy - MRT hippocampal asymmetry;

- $\quad$ seizure trigger factor - sleep deprivation;

- no epilepsy family history and history of febrile seizures;

- no concomitant diseases;

- focal neurological symptoms - intention tremor and dysdiadochokinesia for the left arm, brisk right patellar and Achilles reflexes;

- $\quad$ serum blood level after 3 months of LEV treatment $12.8 \mathrm{mg} / \mathrm{l}$ (reference limits 10-40 mg/l)

- Intermediate chronotype (50 points), determined by the Morningness-Eveningness Questionnaire (MEQ);

- Melatonin serum levels after 3 months of LEV treatment - at 3 a.m. - $22.00 \mathrm{pg} / \mathrm{ml}$ and at 8 a.m. - $23.60 \mathrm{pg} / \mathrm{ml}$ (reference limits for the Bulgarian population: 3 a.m. - $18.5-180 \mathrm{pg} / \mathrm{ml}$, at 8 a.m. - 3.83-80.4 pg/ml) [12].

- At baseline and after 3 months of LEV treatment:

- normal background with no pathological EEG activity;

- normal background with no pathological EEG activity from nocturnal lab polysomnography (PSG);

- normal sleep architecture from nocturnal lab PSG;

- normal Epworth sleepiness score ( 5 at baseline and 3 after 3 months of therapy).

$\circ \quad$ at baseline with mean sleep latency for all 4 naps from Multiple sleep latency test (MSLT) 11.88 min. and reached $\mathrm{N}_{1}$ stage; after 3 months of LEV treatment with mean sleep latency for all 4 naps 17.75 (5.87 min. prolonged) and reached again $\mathrm{N}_{1}$ stage.

- The patient reported no seizures after LEV initiation.

\section{Discussion}

Patients with epilepsy in general suffer from poor sleep quality due to epileptiform activity during sleep, which disturbs the sleep-wake cycle and leads to excessive daytime sleepiness. Our case, however, shows normal sleep architecture and absence of objective and subjective daytime sleepiness (PSG, MSLT, ESS results). He demonstrates intermediate chornotype (MEQ results), which is not associated with extreme late bed time or extreme early wake time. Melatonin serum levels are in reference limits, but there is no normal night peak concentration. This abnormality may be associated with a later night peak melatonin concentration due to epilepsy impact in the absence of epileptiform activity or sleep architecture disruption. A possible explanation may be the treatment with levetiracetam. Such data has not been reported in literature by now.

\section{Conclusion}

We consider the presented clinical case of special interest because of the combination of absence of normal night peak melatonin concentration, normal sleep parameters and non-extremely presented chronotype. We suggest that such patients could benefit from add-on therapy with melatonin. 


\section{Compliance with ethical standards}

\section{Disclosure of conflict of interest}

We declare no conflict of interest.

\section{Statement of informed consent}

Informed consent was obtained from all individual participants included in the study.

\section{References}

[1] Bazil CW. Sleep and epilepsy. Semin Neurol. 2002; 22: 321-7.

[2] Mendez M, Radke RA. Interactions between sleep and epilepsy. J Clin Neurophysiol. 2001; 18: 106-27.

[3] De Weerd A, de Haas S, Otte A, Kasteleijn-Nolst Trenite D, van Erp G, Cohen A, de Kam M, van Gerven J. Subjective sleep disturbance in patient with partial epilepsy: a questionnaire-based study on prevalence and impact on quality of life. Epilepsia. 2004; 45: 1397-404.

[4] Baldy-Moulinier, M. Temporal lobe epilepsy and sleep organisation. In: M. P. Sterman, M. N. Shouse and P. Passouant (Eds) Sleep and Epilepsy. Academic Press, New York. 1982; 347-359.

[5] Hofstra WA, van der Palen J, de Weerd AW. Morningness and eveningness: when do patients take their antiepileptic drugs? Epilepsy Behav. 2012; 23(3): 320-323.

[6] Kendis H, Baron K, Schuele S. Chronotypes in Patients with Epilepsy: Does the Type of Epilepsy Make a Difference? Drug saf. 2013 Apr; 36(4): 207-15.

[7] Manni R, Cremascoli R, De Icco R, et al. Chronotype in patients with epilepsy: A controlled study in 60 subjects with late-onset focal epilepsy. Epilepsy Behav. September 2015; 50: 1-6.

[8] Brezinski A. Melatonin in humans. N Engl J Med 1997; 336(3): 186-195.

[9] Wurtman R. Melatonin. Encyclopedia of Dietary Supplements. 2005; 457-466.

[10] Vishnoi S, Raisuddin S, Parvez S. Glutamate Excitotoxicity and Oxidative Stress in Epilepsy: Modulatory Role of Melatonin; J Environ Pathol Toxicol Oncol. 2016; 35(4): 365-374.

[11] Srinivasan V, Gobbi G, Samuel D, et al. Melatonin: Therapeutic Value and Neuroprotection; 1st Edition; CRC Press Pub. 15 October 2014; 421-23.

[12] Terzieva D, Mateva N, Vladimirova-Kitova L. Melatonin reference limits at 3:00 AM and 8:00 AM in healthy adults. Clin. lab. 2009; 55 (9-10): 359-61. 